\title{
Saikosaponin A modulates remodeling of Kv4.2-mediated A-type voltage-gated potassium currents in rat chronic temporal lobe epilepsy
}

This article was published in the following Dove Press journal:

Drug Design, Development and Therapy

\author{
Yu Hong ${ }^{1,2, *}$ \\ Ning Dengl,* \\ Han-Na Jin ${ }^{3}$ \\ Zheng-Zheng Xuan ${ }^{4}$ \\ Yi-Xiao Qian' \\ Zhi-yong $\mathrm{Wu}^{1,2}$ \\ Wei Xie ${ }^{1,2}$ \\ 'Department of Traditional Chinese \\ Medicine, School of Traditional \\ Chinese Medicine, Southern Medical \\ University, Guangzhou, China; \\ ${ }^{2}$ Department of Traditional Chinese \\ Medicine, Nanfang Hospital, Southern \\ Medical University, Guangzhou, \\ China; ${ }^{3}$ Department of Internal \\ Neurology, People's Hospital of \\ Huizhou Zhongkai Hi-tech Industrial \\ Development Zone, Huizhou, \\ China; ${ }^{4}$ Neuroelectrophysiological \\ Examination Room, Traditional \\ Chinese Medicine Hospital of \\ Guangdong Province, Guangzhou, \\ China \\ *These authors contributed equally \\ to this work
}

Background: Chronic temporal lobe epilepsy (cTLE) is the most common intractable epilepsy. Recent studies have shown that saikosaponin A (SSa) could inhibit epileptiform discharges induced by 4 action potentials and selectively increase the transient inactivating $\mathrm{K}^{+}$currents $\left(I_{\mathrm{A}}\right)$. However, the mechanisms of SSa on $I_{\mathrm{A}}$ remain unclear. In this study, we comprehensively evaluated the anticonvulsant activities of SSa and explored whether or not it plays an antiepileptic role in a Li-pilocarpine induced epilepsy rat model via remodeling Kv4.2-mediated A-type voltage-gated potassium currents (Kv4.2-mediated $I_{\mathrm{A}}$ ).

Materials and methods: All in vitro spontaneous recurrent seizures (SRS) were recorded with continuous video monitoring. Nissl's staining was used to evaluate the SSa protection of neurons and immunohistochemistry, Western blot, and quantitative reverse transcription PCR were used to quantify the expression of Kchip1 and Kv4.2 in the hippocampal CA1 field and the adjacent cortex following Li-pilocarpine induced status epilepticus. We used whole-cell current-clamp recordings to evaluate the anticonvulsant activities of SSa in a hippocampal neuronal culture model of cTLE, while whole-cell voltage-clamp recordings were used to evaluate the modulatory effects of SSa on Kv4.2-mediated $I_{\mathrm{A}}$.

Results: SSa treatment significantly reduced the frequency and duration of SRS over the course of eight weeks and increased the production of Kchip1 and Kv4.2. In addition, SSa attenuated spontaneous recurrent epileptiform discharges (SREDs) in the hippocampal neuronal model and up-regulated Kv4.2-mediated $I_{\mathrm{A}}$.

Conclusions: SSa exerted a disease-modifying effect in our cTLE rat model both in vivo and in vitro; the increase in Kv4.2-mediated $I_{\mathrm{A}}$ may contribute to the anticonvulsant mechanisms of SSa.

Keywords: saikosaponin A, epilepsy, pilocarpine, $\mathrm{Mg}^{2+}$-free, Kchip1, Kv4.2-mediated $I_{\mathrm{A}}$

\section{Background}

Epilepsy is a brain disorder characterized by an enduring predisposition to epileptic seizures, as well as by the neurobiologic, cognitive, psychological, and social consequences of this condition. ${ }^{1}$ One epidemiological survey estimated that there are $\sim 70$ million epileptic patients in the world. ${ }^{2}$ Approximately one-third of the epileptic population has chronic temporal lobe epilepsy (cTLE), which is a common type of acquired epilepsy (AE), and accounts for $70 \%$ of refractory epilepsies. ${ }^{3}$ The repeated attacks not only reduce quality of life, but also cause serious psychological and economic burdens. ${ }^{4}$ Traditional antiepileptic drugs cannot fully control seizures and 40\% of patients show drug resistance. ${ }^{5}$ Therefore, the search for new effective antiepileptic medications is urgent.
Correspondence: Wei Xie School of Traditional Chinese Medicine, Southern Medical University, Guangdong 5I05I5, China

Tel +862061641888

Email xieweizn@126.com 
The pathogenesis of AE has not yet been thoroughly elucidated. In recent years, research findings have implicated that an abnormal construction and function of ion channels may contribute to cTLE. ${ }^{6,7}$ Voltage-gated potassium (Kv) channels are a large family of channels divided into the Kv1-Kv4 subfamilies. ${ }^{8}$ In hippocampal CA1 pyramidal neurons, most of the subthreshold-operating somatodendritic transient outward potassium channels, those with A-type $\mathrm{K}^{+}$current (also known as $I_{\mathrm{A}}$ ), are regulated by $\mathrm{Kv}$-pore-forming subunits (Kv4 channels). ${ }^{4,9}$ These channels are involved in the regulation of back-propagating action potentials (bAPs) in neuronal somadendrites, controlling the neuronal cell excitation level and discharge frequency. ${ }^{10-12}$ Disruptions in these important Kv4 channels may therefore result in an increased propensity to develop seizures. ${ }^{13}$ Numerous studies have attempted to link Kv4.2, a key and principle pore-forming subunit within the Kv4 subfamily of voltage-gated potassium channels, especially in the brain, to patients with cTLE. Such patients have been shown to have dysfunctional Kv4.2, thereby increasing the susceptibility of suffering from epileptic seizures (ES). ${ }^{14,15}$ Different auxiliary proteins play important roles in channel trafficking and function. ${ }^{16}$ For instance, neuronal calcium sensor-1 (NCS-1) may connect with the Kv4 channel alpha-subunits to regulate expression, ${ }^{17}$ and Kv4.2 is a target of the ERK pathway in the induction of status epilepticus (SE) ${ }^{18} \mathrm{Kv} 4.2$ channel function may also be influenced by pituitary adenylate cyclase activating polypeptide and lead to the downstream activation of PKA and ERK1/2. ${ }^{19}$ Recent research has focused on the $\mathrm{K}^{+}$channel-interacting proteins (Kchips), which can regulate the $\mathrm{Kv} 4$ channel by modifying its expression level and intrinsic biophysical properties. ${ }^{20}$

Saikosaponin A ( $\mathrm{SSa}$ ) is a triterpene saponin (chemical structure shown in Figure 1A) derived from the medicinal plant Bupleurum chinensis DC (Umbelliferae). ${ }^{21}$ In our previous studies, we showed that SSa acts as an anticonvulsant and anti-epileptic in both the pentylenetrazole kindling model and the maximal electroshock-induced seizure model. ${ }^{22-24}$ This effect may occur through the inhibition of glial fibrillary acidic protein expression and the activation of rat hippocampal astrocytes induced by glutamate, or otherwise by decreasing the $\mathrm{Ca}^{2+}$ levels and the secretion of IL- 6 from glutamate-activated rat hippocampal astrocytes. ${ }^{25}$ Recent studies have shown that SSa inhibits spontaneous recurrent epileptiform discharges (SREDs) induced by applying a $\mathrm{Mg}^{2+}$-free solution in hippocampal neuronal cultured models of AE. It may also restrain the NMDA-evoked current and the peak current amplitude of $I_{\mathrm{NaP}} \cdot{ }^{26}$ Another study suggested that SSa may inhibit the epileptiform discharges induced by
4 action potentials (AP) and enhance $I_{\mathrm{A}} \cdot{ }^{27}$ Nevertheless, the underlying mechanisms by which SSa modulates the transient outward potassium channel and affects epileptiform activity have not been fully elucidated. In particular, its anti-epileptic effects in vivo have yet to be reported.

In the present study, we aimed to comprehensively explore the anti-epileptic capacity of SSa and its underlying anticonvulsant mechanisms in an epilepsy rat model. We used the Li-pilocarpine induced refractory epilepsy rat model of cTLE in vivo and the $\mathrm{Mg}^{2+}$-free hippocampal neuronal culture (HNC) model of acquired epilepsy in vitro. Spontaneous recurrent seizures (SRS) were recorded by continuous video monitoring and the seizure frequency and duration in a time frame of 3 days were recorded by authors watching the monitoring videos. Nissl's staining was used to evaluate the protection of SSa on neurons, and immunohistochemistry (IHC), Western blot (Wb) and RT-qPCR were used to quantify the expressions of Kchip1 and Kv4.2 proteins and the mRNA levels in the hippocampi CA1 region and the adjacent cortex. Whole-cell voltage-clamp recordings were used to evaluate the modulation of SSa on epileptiform discharges and Kv4.2-mediated A-type $\mathrm{K}^{+}$currents. Our findings suggest that SSa can be an effective candidate for the treatment of cTLE.

\section{Materials and methods Reagents and antibodies}

Saikosaponin A (purity $>98 \%$, obtained from Shanghai Yuanye Bio-Technology Co, Ltd, Shanghai, China), was dissolved in DMSO; the final DMSO concentration did not exceed $0.1 \%$ throughout the study. Neurobasal-A medium, B27, fetal bovine serum, and horse serum were obtained from Gibco-BRL (Invitrogen Corp., San Diego, CA, USA). Recordings were performed in the presence or absence of $\mathrm{SSa}$ or the vehicle. Antibodies specific to Kchip1 and Kv4.2 were obtained from Abcam (Cambridge, MA, USA). Other reagents were purchased from Sigma (St Louis, MO, USA).

\section{Status epilepticus induction} In vivo study

Male Wistar rats, weighing between 220 and $250 \mathrm{~g}$ at the beginning of the experiment ( $\mathrm{n}=102)$, were purchased from the Southern Medical University, Guangzhou, China. The animals were housed and handled in strict accordance with the guidelines of the institutional and national Committees of Animal Use and Protection. Rats were housed at a temperature of $23^{\circ} \mathrm{C}$ to $25^{\circ} \mathrm{C}$ and a humidity of $45 \%$ to $55 \%$ in a controlled environment with a 12/12-hour modified 
A

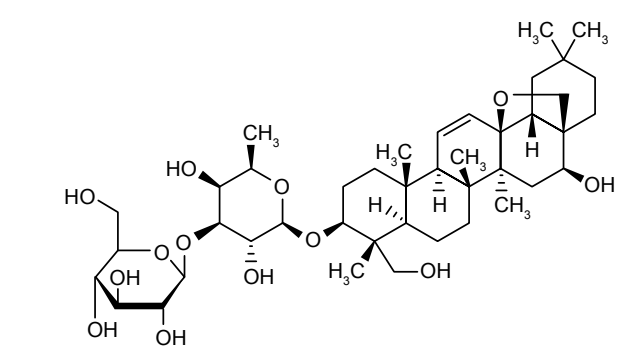

C
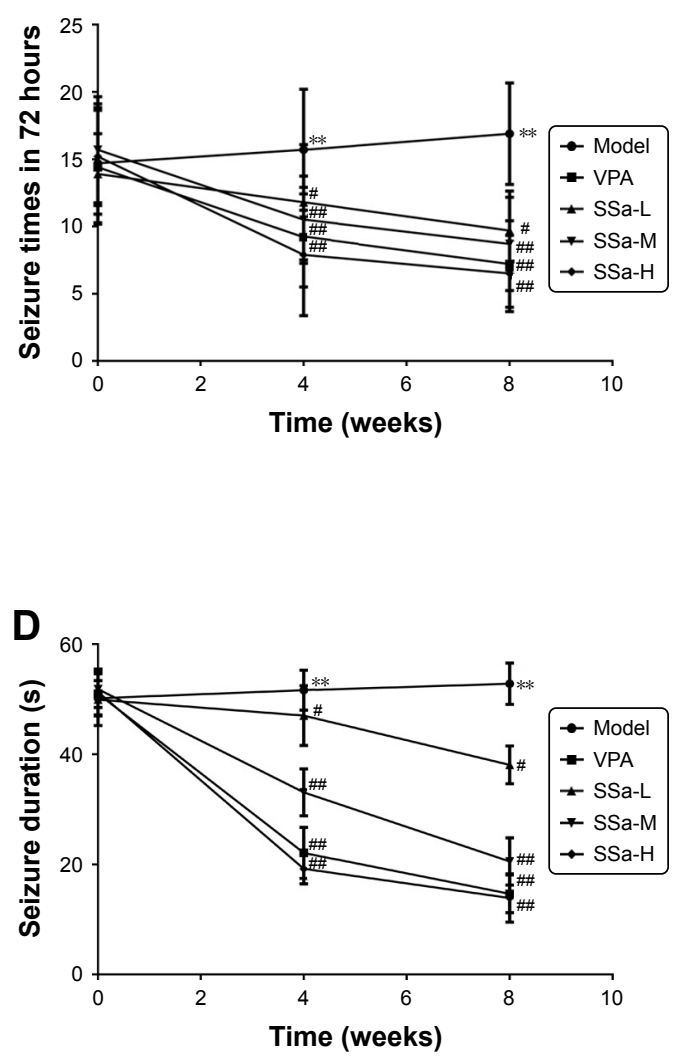

B

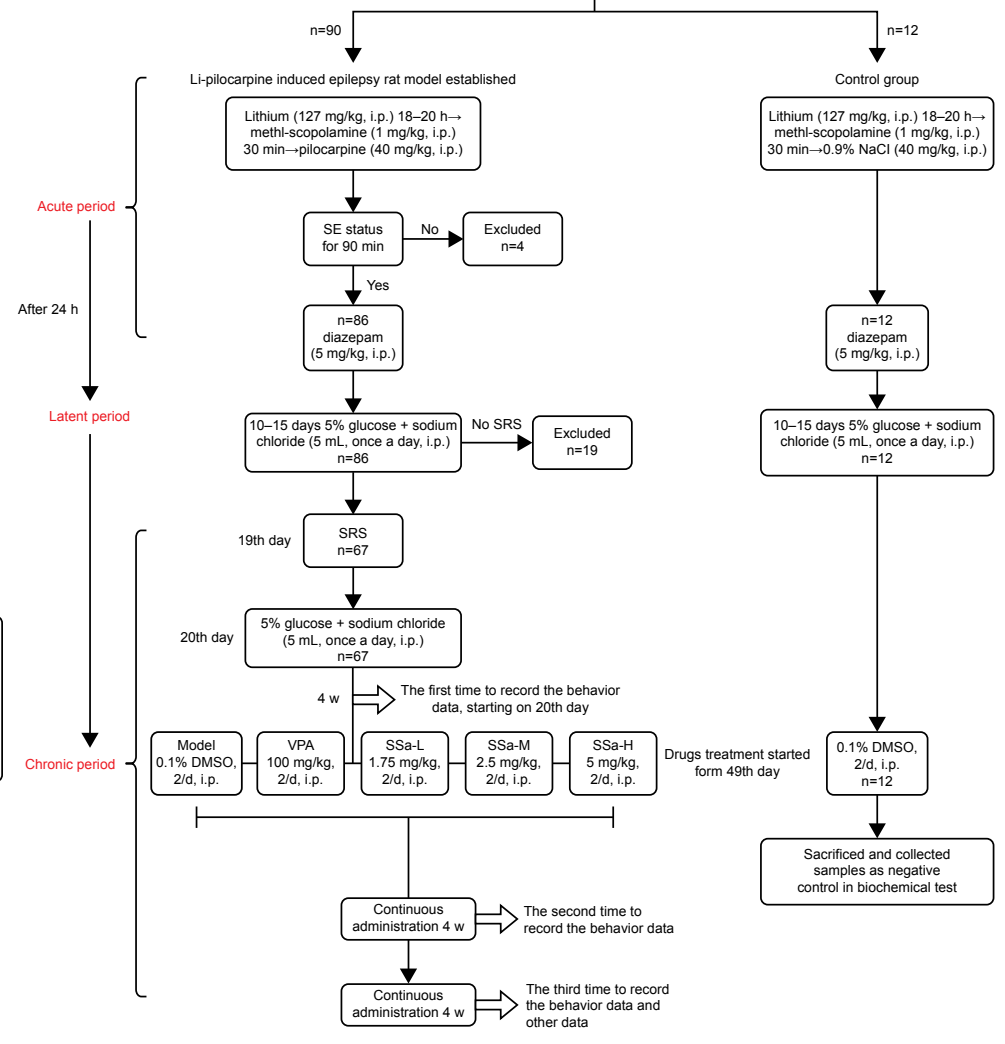

E

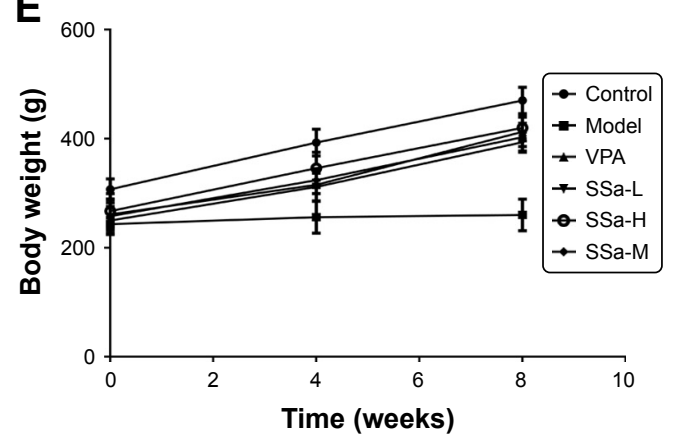

Figure I Effects of SSa on epilepsy behaviors in Li-pilocarpine induced SRS.

Notes: (A) Chemical structure of saikosaponin a (SSa). (B) Flow chart of establish Li-pilocarpine induced epilepsy rat model and drugs treatment allocated. (C) Effect of SSa on seizure frequency. (D) Effect of SSa on seizure duration. (E) Effect of SSa on body weights. Data are expressed as means \pm SD. ${ }^{* *} p<0.0 \mathrm{I}$ vs control; ${ }^{\#} p<0.05$, $p<0.0 \mathrm{I}$ vs model.

Abbreviations: SRS, spontaneous recurrent seizures; SSa-H, high saikosaponin A; SSa-L, low saikosaponin A; SSa-M, medium saikosaponin A; VPA, valproic acid.

dark-light cycle (light from 7:00 am to 7:00 pm). This protocol was approved by the Committee of the Ethics of Animal Experiments of Southern Medical University (Permit Number: SCXK 2011-0015).

In our model, 90 rats were intraperitoneally (i.p.) injected with lithium $(127 \mathrm{mg} / \mathrm{kg}) 18-20$ hours prior to the administration of pilocarpine (40 $\mathrm{mg} / \mathrm{kg}$, i.p.). In order to prevent peripheral cholinergic effects, animals received the cholinergic antagonist methyl-scopolamine (1 mg/kg, i.p.)
30 minutes before pilocarpine injection. After 90 minutes of SE establishment, animals received diazepam $(5 \mathrm{mg} / \mathrm{kg}$, i.p.) to mitigate the seizures. The assessment of behavioral seizures was based on the Racine limbic seizures classifica$\operatorname{tion}^{28}$ and the SE criteria were measured from the time when the animal began to present sustained behaviors or higher limbic seizures of scores IV or $\mathrm{V}$, with no spontaneous recovery. ${ }^{29-31}$ To avoid false-positive results, animals that had not reached SE or that did not spontaneously recovery were 
excluded from this study. The remaining 12 rats were control rats and were treated with lithium, methyl-scopolamine, and diazepam, just like the experimental rats, but with $0.9 \% \mathrm{NaCl}$ instead of pilocarpine. During the latent period, after the acute exacerbation, the rats were injected with 5\% glucose and sodium chloride ( $5 \mathrm{~mL}$, once a day, i.p.). When rats had entered the chronic phase, drug administration started.

\section{Drug treatments}

SRS appeared in the chronic period and the behavior of the rats was continuously monitored after SE, using a digital camera with night vision (Ying Bao Intelligent Equipment Co., Ltd, Guangzhou, China). We compared the seizure counts before treatment, and during the 4 or 8 consecutive weeks of treatment. SRS rats were divided into five groups of twelve animals each: model group, valproic acid (VPA) group, low SSa (SSa-L) group, moderate SSa group (SSa-M) group, high SSa (SSa-H) group, and one control group. The rats in the model and control groups underwent intraperitoneal injections of $0.1 \%$ DMSO twice a day. In the VPA group rats received $100 \mathrm{mg} / \mathrm{kg}$ VPA twice a day by intraperitoneal injection. Rats in the low, moderate, and high SSa groups received $1.75,2.5$, and $5 \mathrm{mg} / \mathrm{kg} \mathrm{SSa}$ twice a day through intraperitoneal injection. All treatment periods lasted 8 weeks. The drugs in the VPA, SSa-L, SSa-M, and SSa-H groups were dissolved in equal amounts of DMSO. The doses of SSa were chosen based on results from our previous experiments ${ }^{27,32,33}$ and preliminary experiments. The details of drug treatment allocated can be found in Figure 1B. In addition, the weights of rats after drug treatments were also recorded.

\section{Subsequent sampling}

After behavior testing, all rats were intraperitoneally injected with $10 \%$ chloral hydrate anesthesia and weighed. The whole brains were harvested from each group of rats after perfusion and fixation with $4 \%$ paraformaldehyde fluid in a $4{ }^{\circ} \mathrm{C}$ refrigerator overnight. After being fixed, brains were dehydrated in $30 \%$ sucrose in PBS, then $30 \mu \mathrm{m}$ frozen sections were cut for the Nissl staining and immunohistochemistry assays. The hippocampus from each rat was isolated on ice, in which the left side was quickly placed in liquid nitrogen to be frozen and was stored at $-80^{\circ} \mathrm{C}$ for protein-related analysis. The right side of the hippocampus was placed in RNA protective liquid of 10 times the hippocampus volume, for the RT-qPCR assay.

\section{Nissl staining}

Nissl staining was applied to observe the extent of neuron loss. At the end of the experiments, the brain tissues of six rats from each group were deeply anesthetized with thionembutal and sequentially perfused through the heart. Frozen coronal sections were then processed for Nissl staining $(30 \mu \mathrm{m})$ using Cresyl violet.

\section{Immunohistochemistry}

At the end of the experiments, the brain tissues of six rats from each group were collected to determine the levels of Kchip1 and Kv4.2, and prepared as previously described. ${ }^{34}$ The paraffin-embedded hippocampal sections were deparaffinized, rehydrated, and pre-treated with hydrogen peroxidase in a phosphate buffer solution. Heat-induced antigen retrieval was then conducted. After blocking with the appropriate antisera, sections were incubated with anti-Kchip1 and anti-Kv4.2 (1:100 dilution). After incubation with HRPconjugated secondary antibody, tyramide amplification, and streptavidin-HRP, positive signals were visualized with a diaminobenzidine kit and counter-stained with hematoxylin.

\section{Western blot}

$\mathrm{Wb}$ was performed at the end of the experiments. The brain tissues of six rats from each group were collected to determine the protein levels of Kchip1 and Kv4.2. Briefly, the protein lysates were loaded onto $10 \%$ SDS-PAGE gel for separation, electrotransferred onto PVDF membranes, and blocked in 5\% nonfat milk in Tris-buffered saline and Tween. The membranes were incubated overnight with anti-Kchip1, anti-Kv4.2 (1:1,000 dilution), and anti-GAPDH (ZSGB-BIO, $1: 10,000)$ at $4^{\circ} \mathrm{C}$. This procedure was followed by incubation with horseradish peroxidase (HRP)-conjugated secondary antibody. Bands were evaluated by densitometry using Molecular Imaging Software Version 5.0 (Kodak 2000MM System). The OD was normalized against that of GAPDH.

\section{Real-time reverse transcriptase PCR}

RT-PCR was performed as previously described. ${ }^{35}$ At the end of the experiments, the brain tissues of six rats from each group were harvested for analysis. Total RNA was extracted from the brain samples using TRIzol reagent (TaKaRa Bio, Dalian, China). The reverse transcriptase reactions were performed on a Stratagene Robocycler Gradient 96 Thermal Cycler (Stratagene, San Diego, CA, USA) using a reverse transcriptase kit (TaKaRa Bio, Dalian, China) according to the manufacturer's instructions. The single-stranded cDNA was amplified through comparative quantitative real-time reverse transcriptase PCR using a SYBR green Master Mix kit (Thermo Fisher Scientific, Waltham, MA, USA) on an Mx3005 (Stratagene). 


\section{In vitro study}

Hippocampal cells were prepared from one-day-old postnatal Wistar rats and the culture and HNC models were established according to the protocol described in our previous studies. ${ }^{26} \mathrm{~A} 3$ hour exposure of $\mathrm{Mg}^{2+}$-free solution to cultured hippocampal neurons was employed to induce SREDs, as previously described, ${ }^{36}$ and was followed by 24 hours of culture maintenance. SRED rats were divided into: model (0.1\% DMSO), VPA (0.5 mM), SSa-L (1.25 $\mu \mathrm{M})$, SSa-M $(2.5 \mu \mathrm{M})$, SSa-H $(5 \mu \mathrm{M})$, and control $(0.1 \%$ DMSO $)$ groups. The neurons in all groups were treated with different drugs for 48 hours.

\section{Patch-clamp recordings}

At the end of the experiments, we collected eight neuron cells from each group to record the discharges and currents. The solution containing the SRED electrodes was prepared as previously described. ${ }^{26}$ The Kv4.2-mediated $I_{\mathrm{A}}$ current recording method with an intracellular solution containing (in $\mathrm{mM}$ ): 140 potassium gluconate, 0.2 EGTA, $3 \mathrm{MgCl}_{2}$, 10 Hepes, 4 MgATP, and $0.5 \mathrm{NaGTP}$ (pH adjusted to 7.2 with $\mathrm{KOH}$ ). The extracellular solution contained (in $\mathrm{mM}$ ): $130 \mathrm{NaCl}, 2.5 \mathrm{KCl}, 1.25 \mathrm{NaH}_{2} \mathrm{PO}_{4}, 26 \mathrm{NaHCO}_{3}, 1 \mathrm{MgSO}_{4}$, $2 \mathrm{CaCl}_{2}$, and 10 glucose, with five tetraethylammonium (TEA) and 500 tetrodotoxin (TTX), except when otherwise noted. Currents were filtered at $5 \mathrm{kHz}$ and sampled at $10 \mathrm{kHz}$. The Kv4.2-mediated $I_{\mathrm{A}}$ was isolated using the prepulse protocol described by subtracting the currents recorded during the depolarizing steps $(\mathrm{Vp}$; from -90 to $+70 \mathrm{mV}$ ) preceded by a 1 seconds prepulse to $-40 \mathrm{mV}$ from the currents obtained during the depolarizing steps preceded by a 1 seconds prepulse to $-110 \mathrm{mV} \cdot$. $^{37,38}$

\section{Data analysis}

Data are presented as mean \pm SD and we used SPSS (version 21) for all statistical analyses. One-way ANOVA was used and $p<0.05$ was considered to be statistically significant.

\section{Results}

\section{Effects of SSa on epilepsy in a Li-pilocarpine-induced rat model}

Clinical signs of seizure activity were observed in every pilocarpine-treated animal. All rats exhibited a well-defined pattern of behavior after treatment, such as cholinergic reactions. ${ }^{39-41} \mathrm{~A}$ total of $95.7 \%$ of the rats experienced SE with bilateral limb clonus, rearing, and falling 15-65 minutes after pilocarpine injection, $78.6 \%$ of which further underwent SRS, which usually occurred $\sim 2$ weeks after the initial SE.
No significant differences in epilepsy behavior were found between any of the groups in the baseline $(p>0.05)$. After the administration of SSa $(1.75,2.5$, or $5 \mathrm{mg} / \mathrm{kg}$ ) for four weeks, every treatment group showed ameliorated epilepsy behaviors in Li-pilocarpine induced SRS, in a dosedependent manner. SSa $(1.75,2.5$, or $5 \mathrm{mg} / \mathrm{kg})$ treatment significantly decreased seizure frequency in pilocarpine-exposed rats $(11.80 \pm 4.289,10.50 \pm 3.240,7.90 \pm 4.532$ vs $15.70 \pm 4.498$, $p<0.05$, Figure 1C). The average seizure duration decreased in the SSa groups $(47.01 \pm 5.433,33.07 \pm 4.254,19.20 \pm 2.740$ vs $51.62 \pm 3.645, p<0.05$, Figure 1D). After SSa administration for eight weeks, seizure frequency was also reduced in the SSa groups $(9.70 \pm 2.946,8.70 \pm 3.466,6.50 \pm 2.838$ vs $16.80 \pm 3.706, p<0.05$, Figure $1 \mathrm{C}$ ) and the duration decreased in the SSa groups $(38.07 \pm 3.427,20.53 \pm 4.294$, $13.90 \pm 4.383$ vs $52.80 \pm 3.735, p<0.05$ Figure $1 \mathrm{D})$. SSa ameliorated epilepsy behavior in a Li-pilocarpine-induced rat model. Furthermore, the weights of rats did not have significant difference before and after different drug treatments (all $p>0.05$; Figure 1E). This suggested that drugs do not affect the weights of rats.

\section{Effects of SSa on neuronal loss in rat hippocampi CAI and the adjacent cortex} Since SSa demonstrated antiepileptic activity in the pilocarpine SRS model, we used Nissl staining to analyze its neuroprotective effects in the hippocampi CA1 region and the adjacent cortex. Figure 2A and B shows neurons with a normal shape and abundant Nissl bodies in the hippocampi CA1 region and the adjacent cortex in the control group. However, the number of Nissl bodies decreased or disappeared, and cell body shrinkage was observed in the model group $(p<0.05)$. These effects were ameliorated after SSa administration $(1.75,2.5$, or $5 \mathrm{mg} / \mathrm{kg})(p<0.05$, Figure $2 \mathrm{C})$.

\section{Effects of SSa on KchipI and Kv4.2 expression in rat hippocampi CAI and the adjacent cortical tissues}

Increased excitability of the CA1 pyramidal neurons in TLE was observed due to the decreased availability of the transient outward potassium channel, mediated by Kv4.2, which is associated with seizure occurrence and progress. ${ }^{42}$ In order to further elucidate the mechanisms behind the SSa modulation of the transient outward potassium channel and its effects on epileptiform activities, we examined the expressions of Kv4.2 and its regulatory protein Kchip1. The immunohistochemistry analysis revealed that Kchip1 and Kv4.2 expression in the hippocampal CA1 field and the adjacent cortex in the model group decreased compared to 
A

Hippocampl CA1
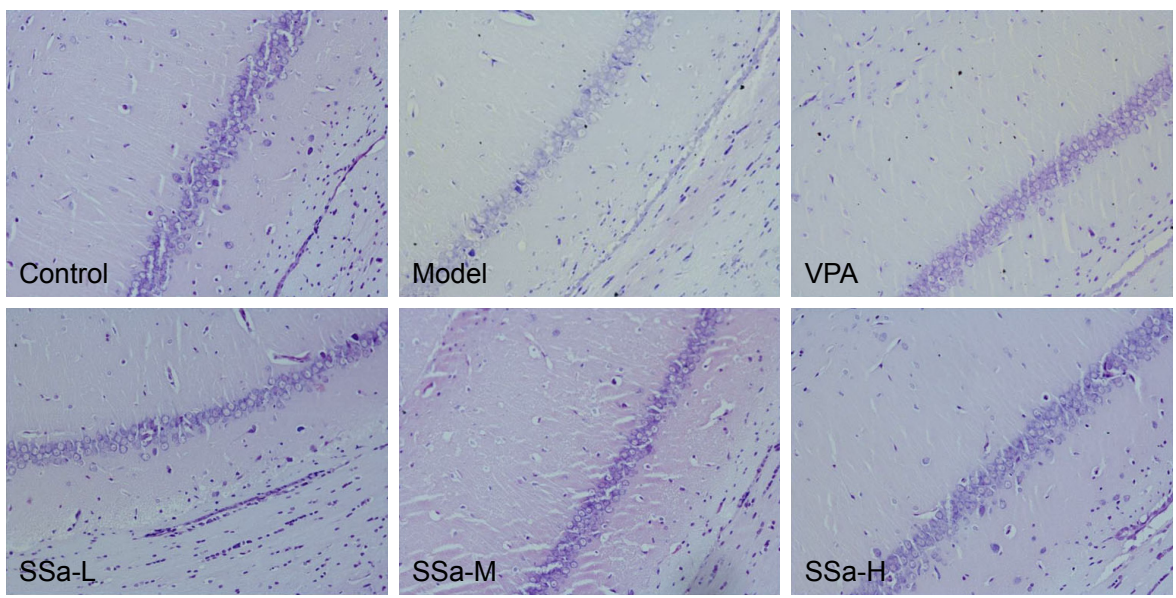

B

\section{Cortex}
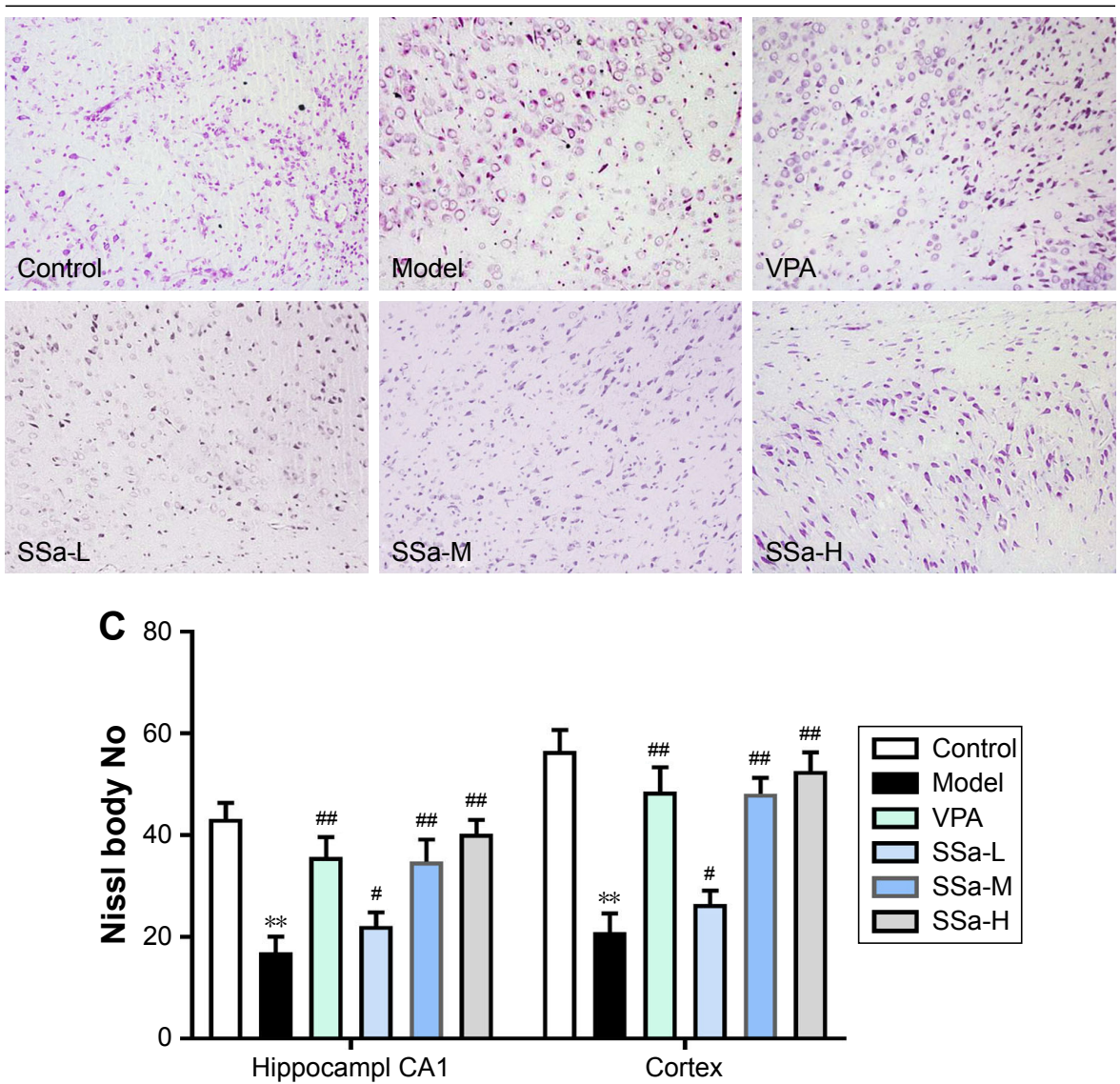

Figure 2 Effects of SSa on neuron loss in Li-pilocarpine induced SRS.

Notes: (A) Nissl staining in the hippocampal CAI region (I200); (B) Nissl staining in the adjacent cortex (I200); (C) Nissl staining analysis in the hippocampal CAI and adjacent cortex. Data are expressed as means $\pm S D$. ${ }^{* *} p<0.0$ I vs control; ${ }^{*} p<0.05,{ }^{\#} p<0.0$ I vs model.

Abbreviations: SSa-H, high saikosaponin A; SSa-L, low saikosaponin A; SSa-M, medium saikosaponin A; VPA, valproic acid.

the control group $(p<0.05)$. This downregulated expression was enhanced by SSa treatment $(p<0.05)$. All of these results were further confirmed by $\mathrm{Wb}$ and RT-PCR analyses $(p<0.05)$ (Figures 3 and 4).

\section{Effects of SSa on SREDs in the HNC} model induced by $\mathrm{Mg}^{2+}$-free solution

The data clearly indicate that SSa abates SRS in vivo, so the logical next step was to determine whether SSa could 


\section{A}
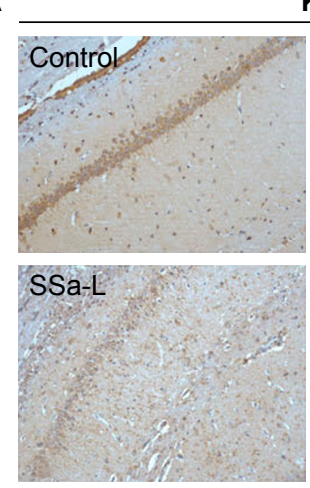

Kchip1-hippocampi CA1

B
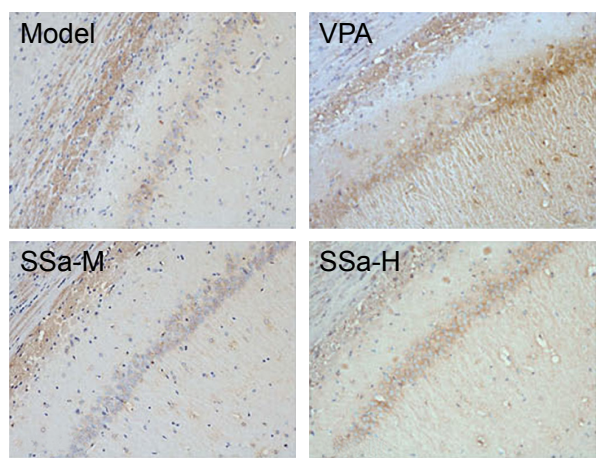

$\mathrm{SSa}-\mathrm{H}$

Kv4.2-hippocampi CA1
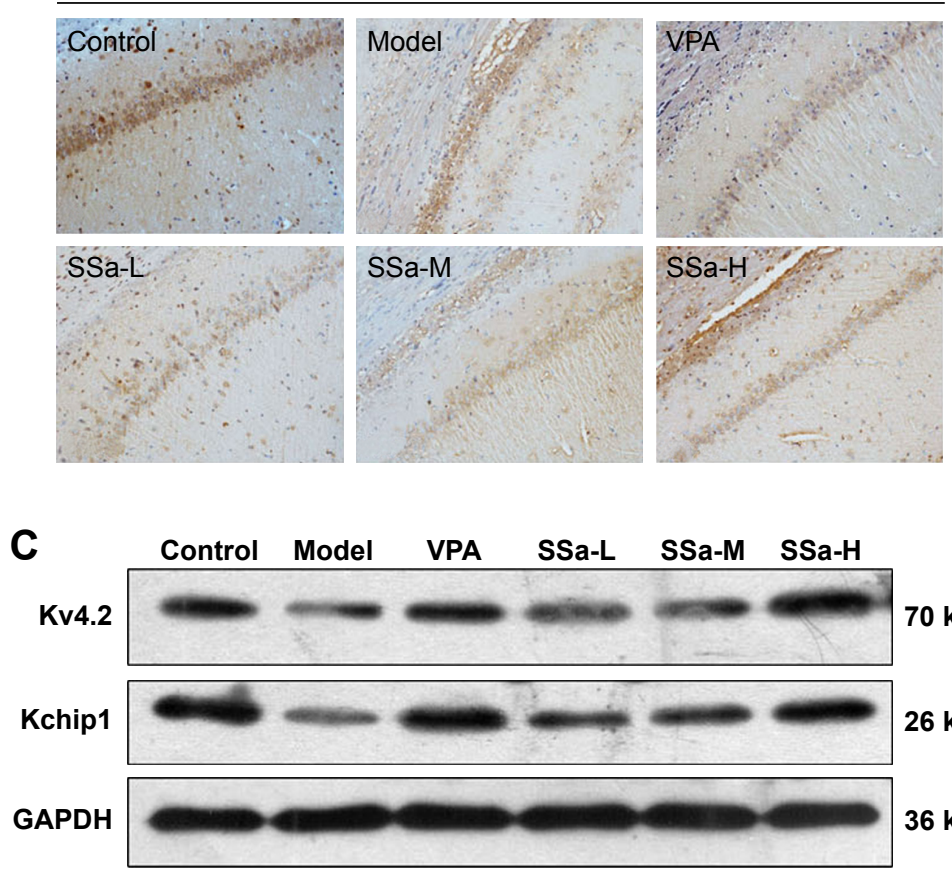

$70 \mathrm{kDa}$

$26 \mathrm{kDa}$

$36 \mathrm{kDa}$
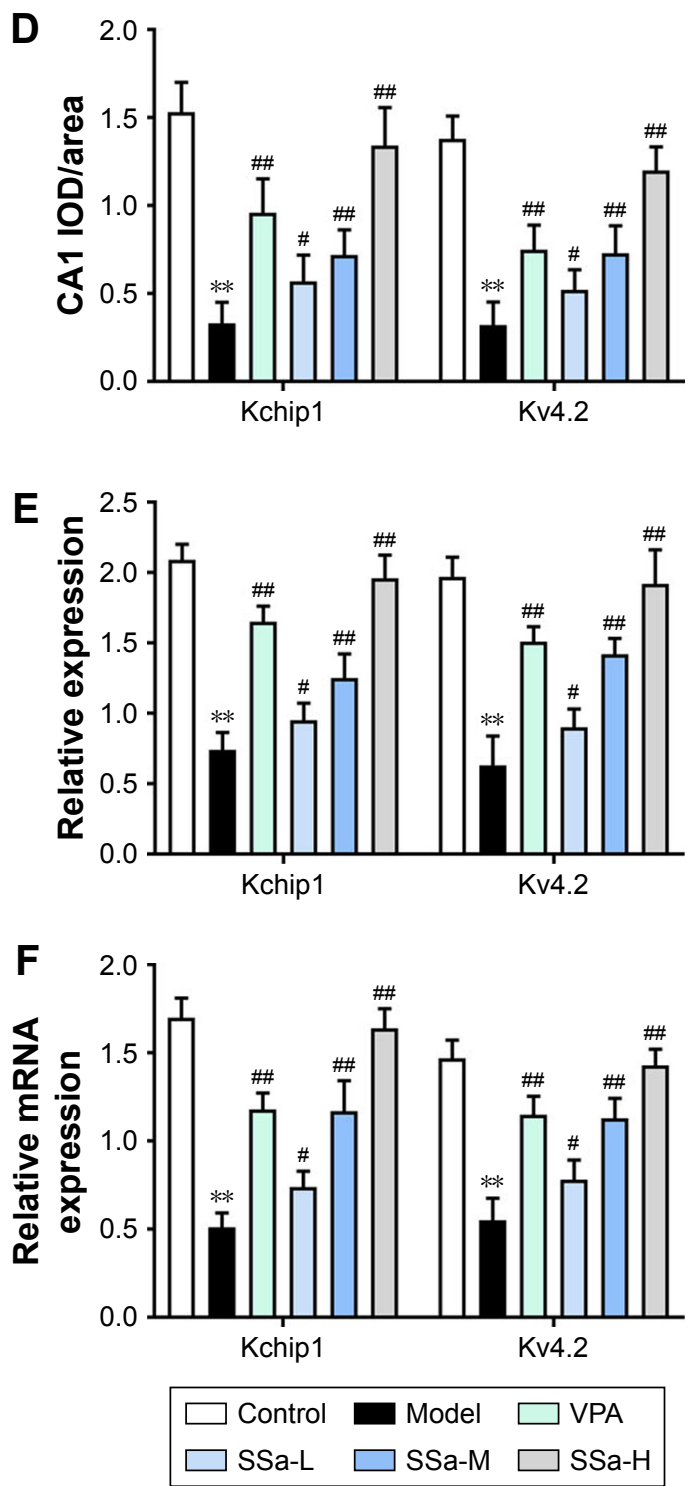

Figure 3 Effects of SSa on Kchipl and Kv4.2 expression in rat hippocampi.

Notes: (A, B, and D) Immunohistochemical staining; (C and E) Western blot analysis; (F) RT-qPCR analysis. Data are expressed as means \pm SD. ** $p<0.0$ I vs control; $\# p<0.05,{ }^{\#} p<0.0$ I vs model.

Abbreviations: SSa-H, high saikosaponin A; SSa-L, low saikosaponin A; SSa-M, medium saikosaponin A; VPA, valproic acid; RT-qPCR, quantitative reverse transcription PCR.

inhibit epilepsy discharges in vitro. To this end, whole-cell current-clamp recordings were carried on the HNC models of cTLE. The anticonvulsant effects of SSa were observed by incubating hippocampal neurons with concentrations of 1.25 , 2.5 , or $5 \mu \mathrm{M} \mathrm{SSa}$ for 48 hours. Interrupted spontaneous action potentials recorded from control neurons were recognized as basic activities. The application of $\mathrm{Mg}^{2+}$-free treated neurons induced SREDs $(p<0.01$, Figure 5A), which indicated that this in vitro model of recurrent epileptic discharges had been successfully established. These discharges were ameliorated by incubation with normal extracellular fluid containing $\mathrm{SSa}(1.25,2.5$, or $5 \mu \mathrm{M})$ for 48 hours, in a dose-dependent manner (Aps: $15.98 \pm 3.391 / \mathrm{s}, 10.54 \pm 3.431 / \mathrm{s}, 3.08 \pm 2.562 / \mathrm{s}$ vs $20.44 \pm 3.270 / \mathrm{s}, p<0.05$, Figure $5 \mathrm{~B}$ ).

\section{Effects of SSa on KchipI and Kv4.2} expression in the HNC model induced by $\mathrm{Mg}^{2+}$-free solution

We observed that SSa exerts an anti-epileptic effect both in vivo and in vitro, and may possibly do so by increasing Kchip1 and Kv4.2 expression. In the in vitro experiments, Kchip1 and Kv4.2 expression in cultured hippocampal neurons decreased after the application of $\mathrm{Mg}^{2+}$-free solution compared with the control group $(p<0.05)$. Significantly increased 
A

Kchip1-cortex
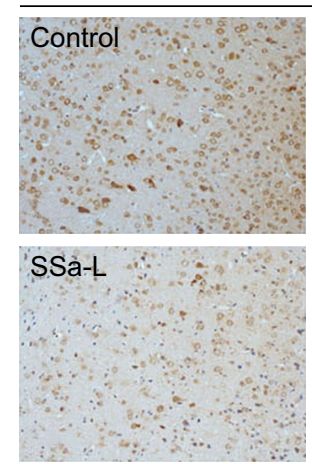

B
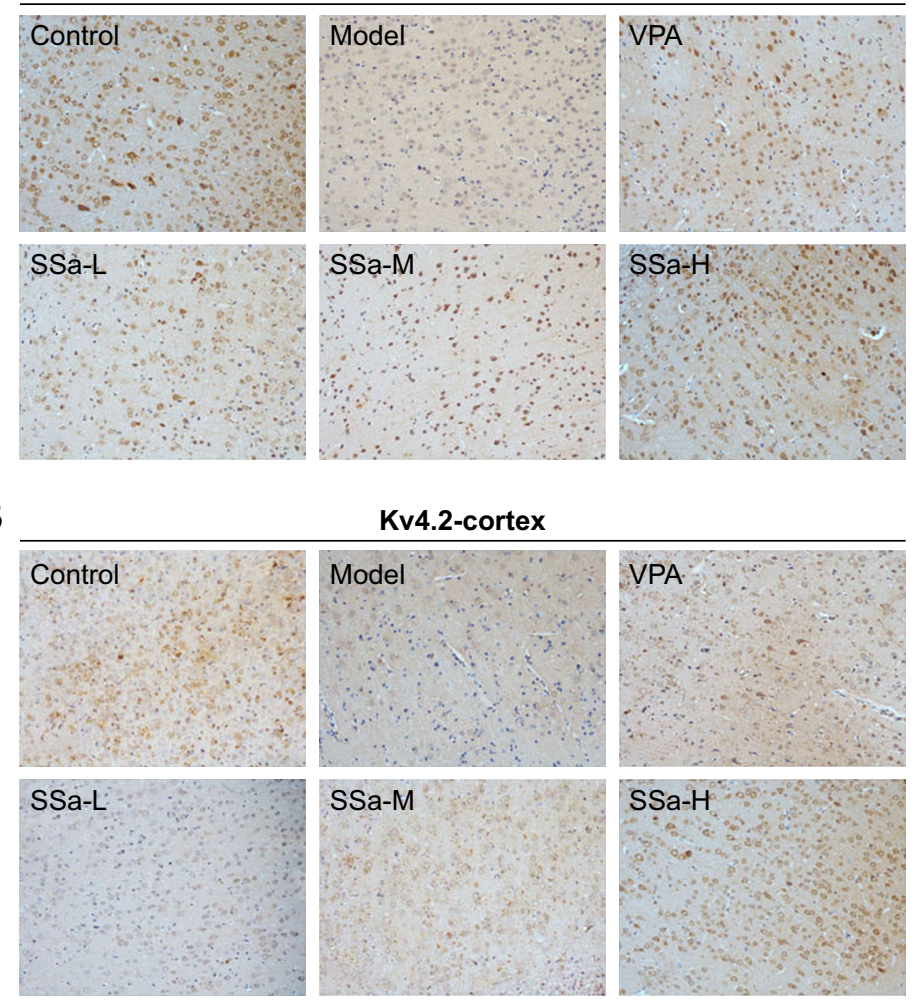

C

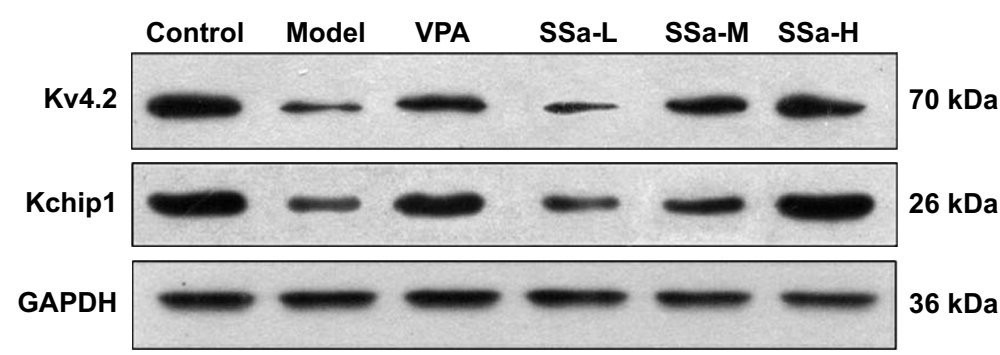

D

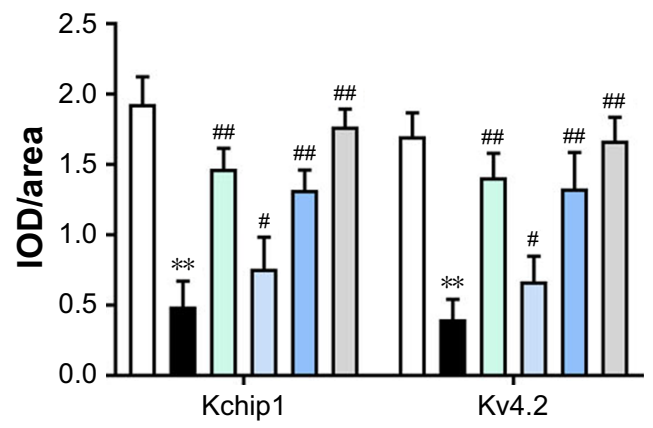

$E$

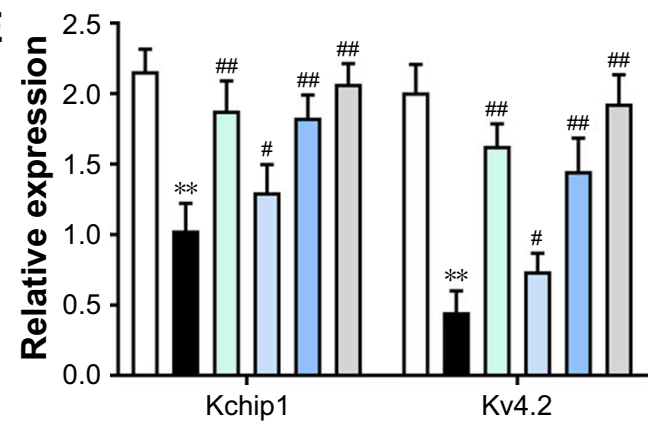

$\mathbf{F}$

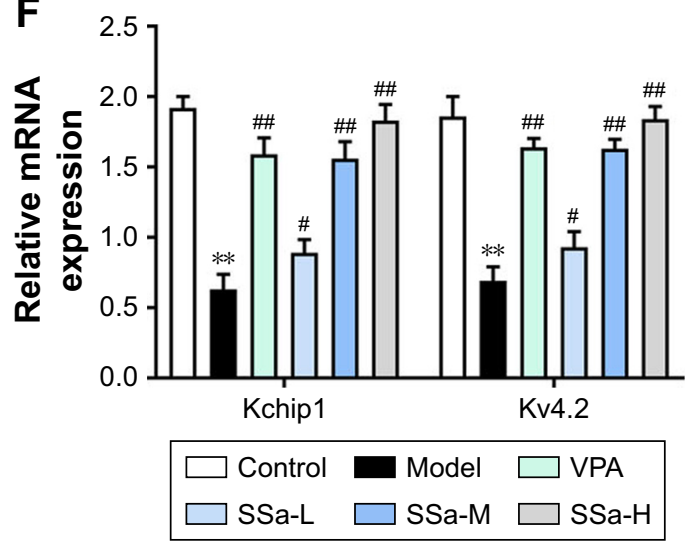

Figure 4 Effects of SSa on Kchipl and Kv4.2 expression in rat adjacent cortex.

Notes: (A, B, and D) Immunohistochemical staining; (C and E) Western blot analysis; (F) RT-qPCR analysis. $\mathrm{n}=6$ animals per group. Data are expressed as means \pm SD. $* * p<0.01$ vs control; $\#<0.05, \# p<0.01$ vs model.

Abbreviations: SSa-H, high saikosaponin A; SSa-L, low saikosaponin A; SSa-M, medium saikosaponin A; VPA, valproic acid; RT-qPCR, quantitative reverse transcription PCR.

expression, however, was witnessed after treatment with $\mathrm{SSa}$ $(1.25,2.5$, and $5 \mu \mathrm{M})(p<0.05$, Figure $6 \mathrm{~A})$. These results were confirmed by $\mathrm{Wb}$ and RT-PCR (Figure 6B and $\mathrm{C}$ ).

\section{Effects of SSa on Kv4.2-mediated $I_{A}$} currents in the HNC model induced by $\mathrm{Mg}^{2+}$-free solution

A previous study showed that $\mathrm{SSa}$ modulates $I_{\mathrm{A}}$ in cultured hippocampal neurons. ${ }^{27}$ We tested the modulatory effect of 48 hours of SSa exposure on $I_{\mathrm{A}}$ in the HNC model. The typical traces of Kv4.2-mediated $I_{\mathrm{A}}$ currents from each group before and after SSa administration are shown in Figure 7A. The Kv4.2mediated $I_{\mathrm{A}}$ current densities significantly decreased after SSa in the $\mathrm{Mg}^{2+}$-free solution induced model group (Figure 7B and $\mathrm{C}$ ), and its statistically significant enhanced effects on the $I$ density $(\mathrm{pA} / \mathrm{pF})$ of Kv4.2-mediated $I_{\mathrm{A}}$ were shown to be voltage dependent $(87.83 \pm 17.609,94.33 \pm 17.134,123.77 \pm 17.066$ vs $67.12 \pm 20.546, p<0.05$, Figure 7D). Additionally, SSa produced a significant change in voltage at half-maximal activation $\left(V_{1 / 2}\right)(23.54 \pm 1.085,16.47 \pm 0.667,13.42 \pm 0.947$ vs $27.26 \pm 2.799, p<0.05$, Figure 7E), which suggests that SSa increased the activity of Kv4.2-mediated $I_{\mathrm{A}}$ currents.

\section{Discussion}

In previous studies, $\mathrm{SSa}$ has been shown to have different pharmacological effects. In the halothane-induced hepatic 
A

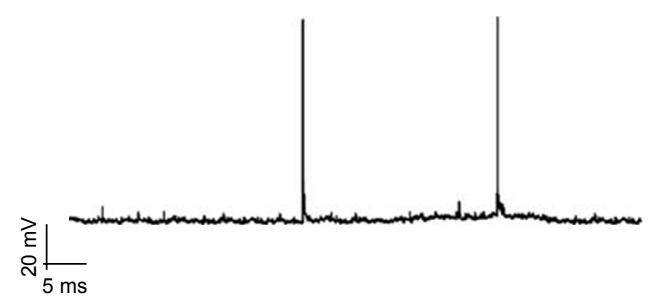

Model

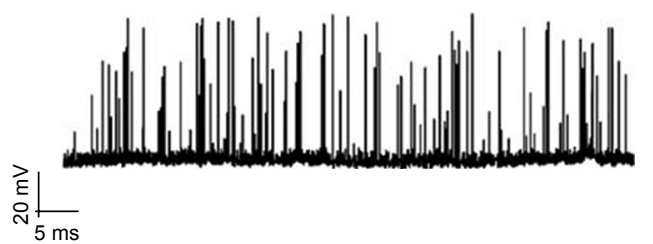

VPA

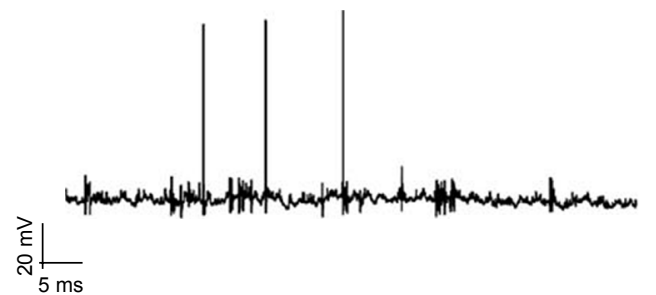

SSa-L

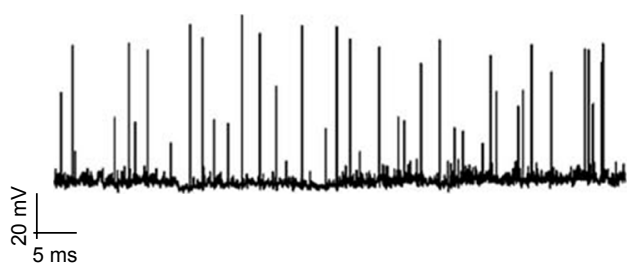

SSa-M

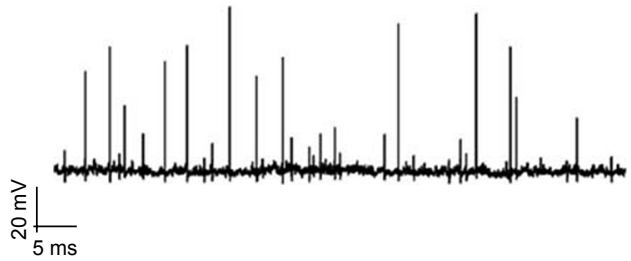

SSa-H

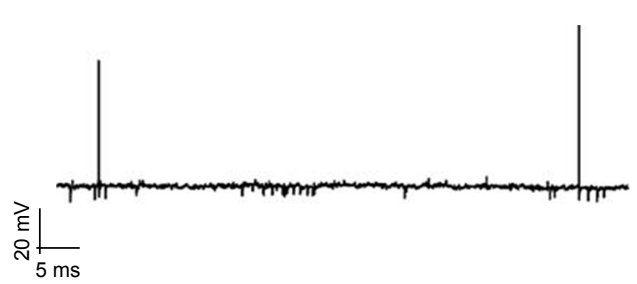

B

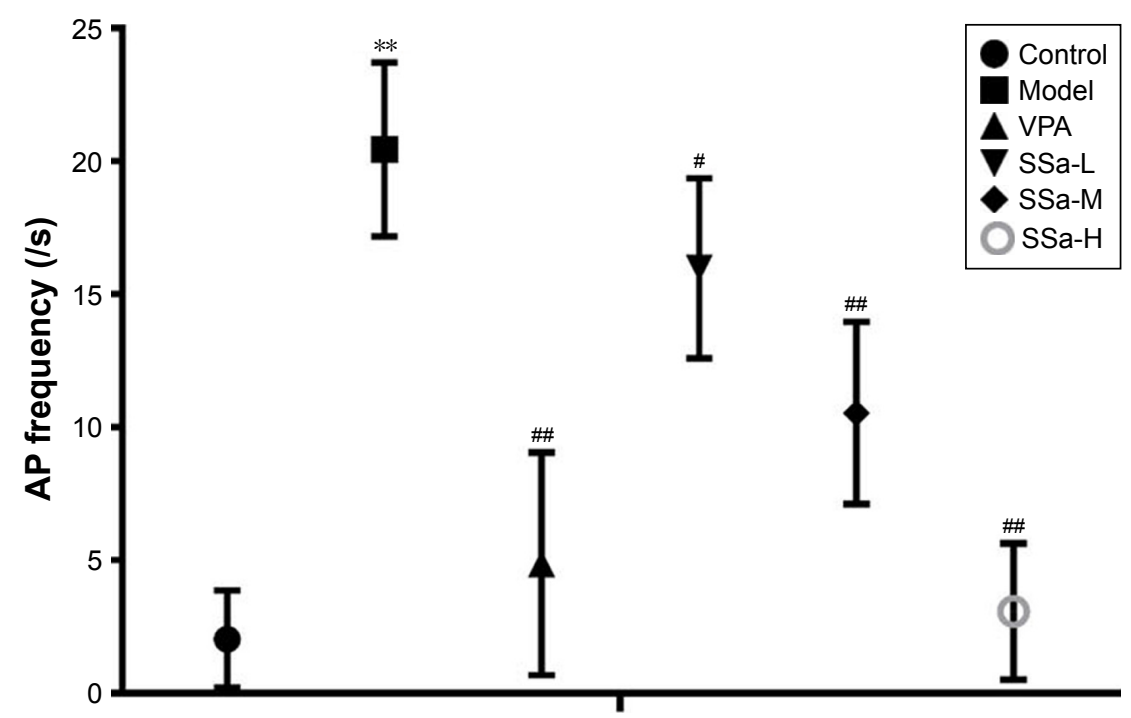

Figure 5 Effects of SSa on SREDs induced by $\mathrm{Mg}^{2+}$-free medium in cultured hippocampal neurons.

Notes: (A) Traces of SREDs; (B) Frequency of $\mathrm{Mg}^{2+}$-free induced SRED analysis. Data are expressed as means \pm SD. $* * p<0.0$ I vs control; ${ }^{*} p<0.05$, $p<0.0$ I vs model. Abbreviations: AP frequency, action potential frequency; SREDs, spontaneous recurrent epileptiform discharges; SSa-H, high saikosaponin A; SSa-L, low saikosaponin A; SSa-M, medium saikosaponin A; VPA, valproic acid.

damage rat model, SSa protected liver cells from damage. ${ }^{43}$ Lu et al suggested that SSa has an anti-inflammatory effect on lipopolysaccharide (LPS)-induced RAW264.7 cells, which was achieved through inhibiting the activated NF- $\mathrm{\kappa B}$ signaling pathway. ${ }^{44}$ In addition, another study determined that
SSa inhibits cell activity and proliferation in human breast cancer, thereby exerting an anti-cancer effect. ${ }^{45}$ However, although some studies have demonstrated that SSa has antiepileptic effect, ${ }^{32,33}$ there is still sparse research about the anti-epileptic effect of SSa on the Li-pilocarpine-induced 


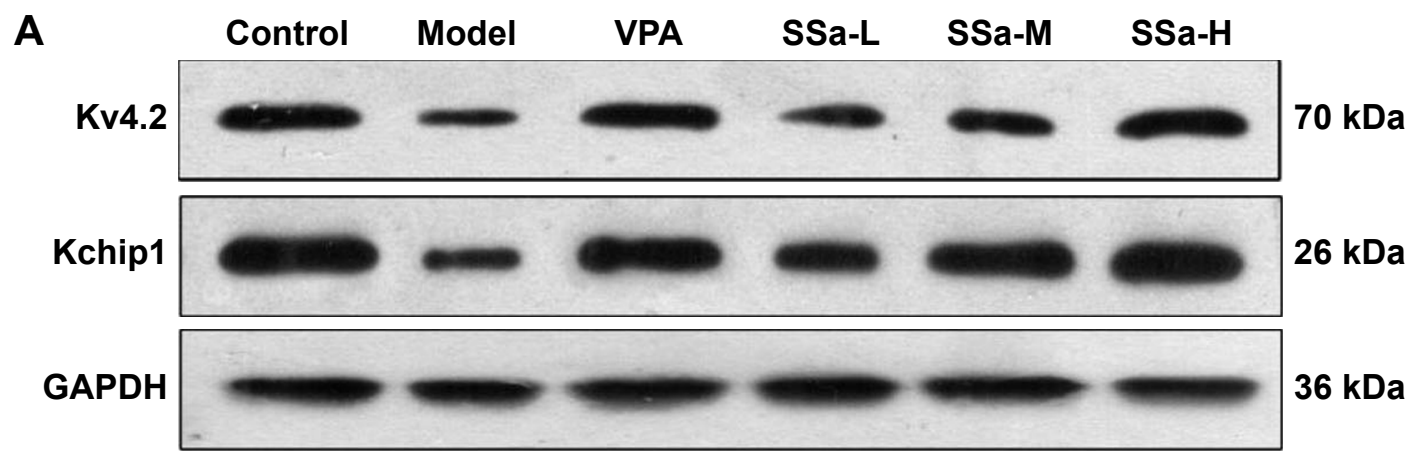

B
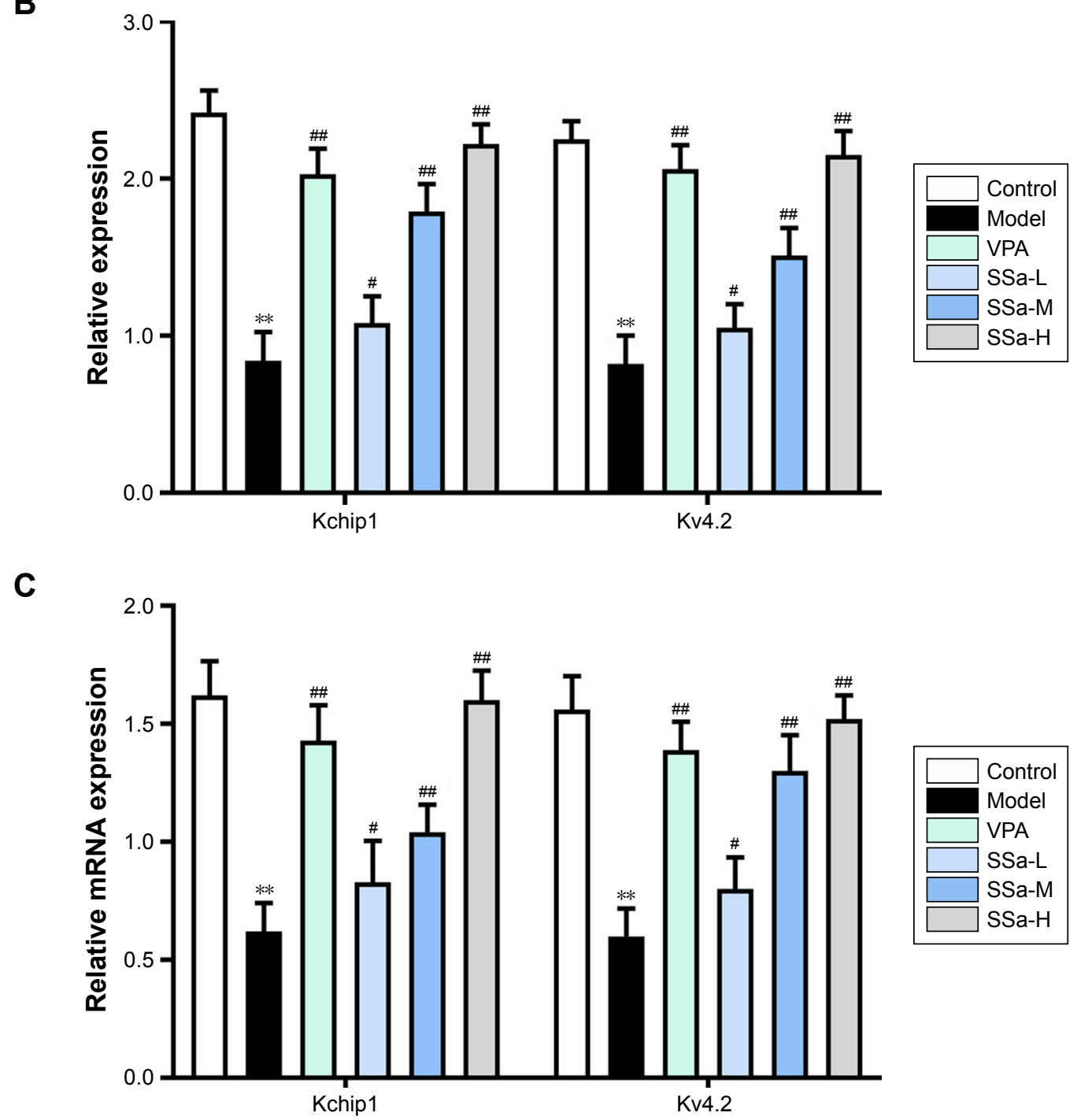

Figure 6 Effects of SSa on Kchipl and Kv4.2 expression in cultured hippocampal neurons.

Notes: (A and B) Western blot analysis; (C) RT-qPCR analysis. Data are expressed as mean \pm SD. ${ }^{* *} p<0.0$ I vs control; ${ }^{\#} p<0.05,{ }^{\#} p<0.0$ I vs model.

Abbreviations: SSa-H, high saikosaponin A; SSa-L, low saikosaponin A; SSa-M, medium saikosaponin A; VPA, valproic acid; RT-qPCR, quantitative reverse transcription PCR.

SRS model. The Li-pilocarpine-induced SRS model is a widely used chronic intractable TLE animal model, ${ }^{46}$ since it mimics many features of the human epileptic condition. ${ }^{47,48}$ It is often used to evaluate the efficacy of antiepileptic activity in rodents. This study is the first to find that SSa treatment had an antiepileptic effect by abating the frequency and duration of seizures in the pilocarpine refractory seizure model, and no deaths or other adverse events were found throughout the course of administration, suggesting that SSa is safe in rats. Similarly, SSa has been shown to inhibit neuronal loss. 
A

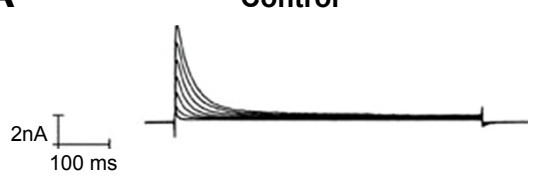

Model

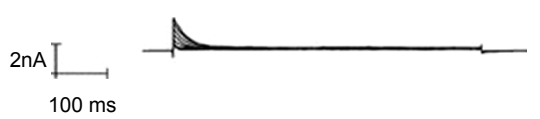

VPA

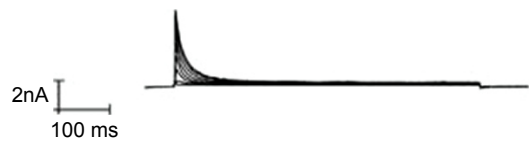

B
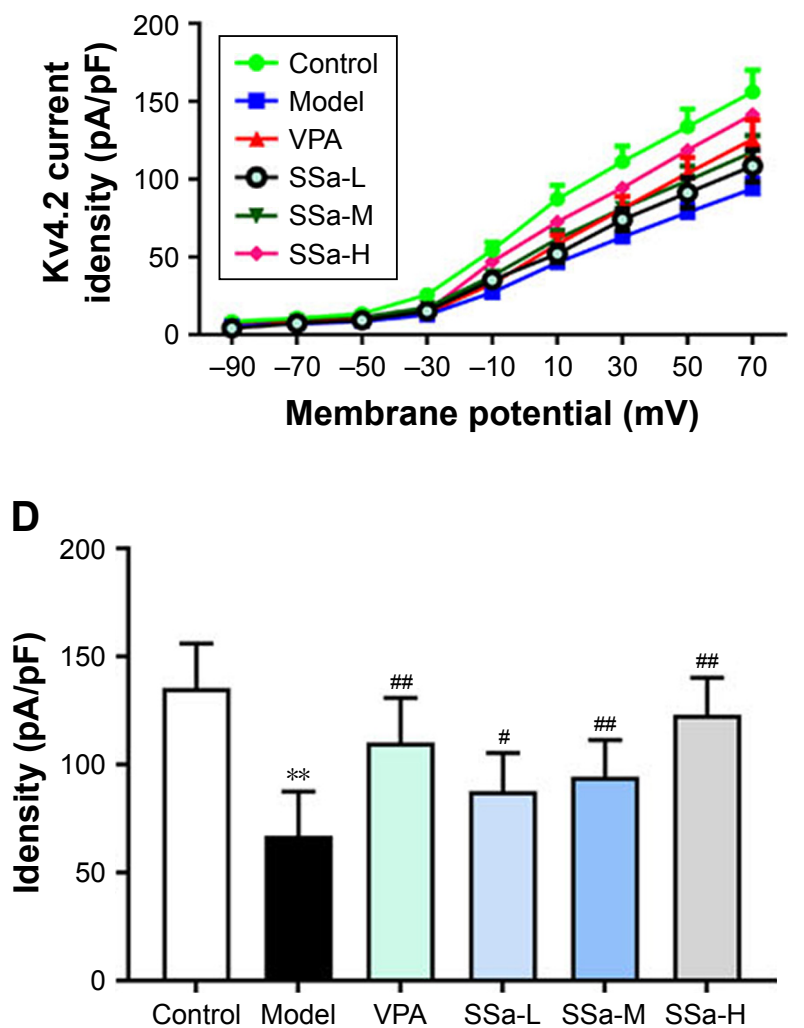

SSa-L

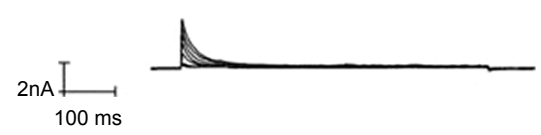

SSa-M

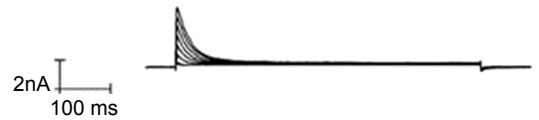

SSa-H

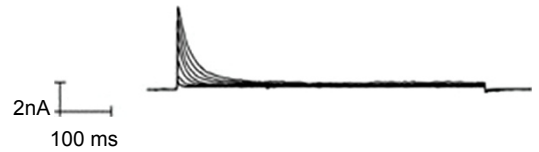

C

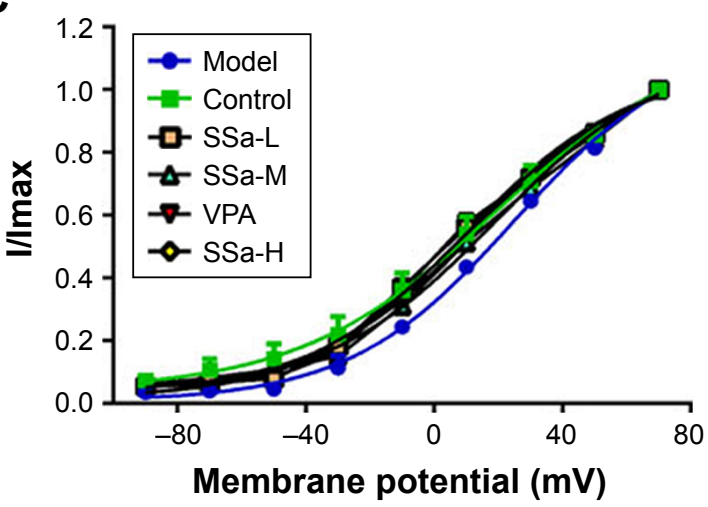

E

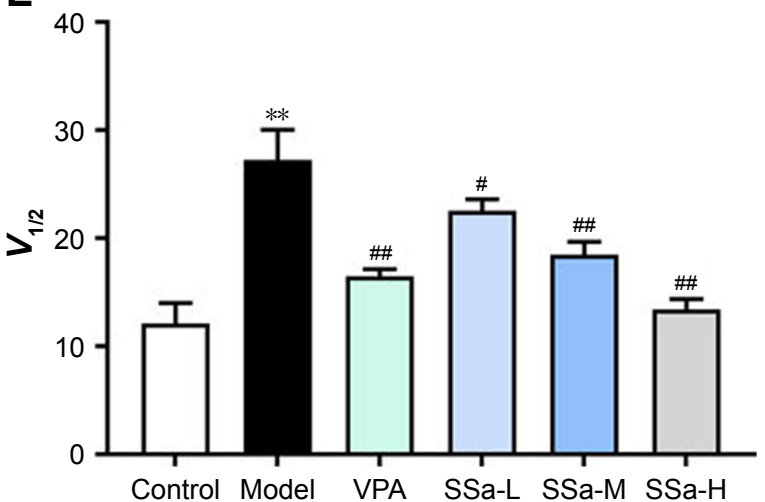

Figure 7 Effects of SSa on Kv4.2-mediated $I_{A}$ currents induced by $\mathrm{Mg}^{2+}$-free medium in cultured hippocampal neurons.

Notes: (A) Traces of Kv4.2-mediated $I_{A}$ currents; (B) $I-V$ curve for Kv4.2-mediated $I_{A}$ currents; (C) Activation curve for Kv4.2-mediated $I_{A}$ currents; (D) Kv4.2-mediated $I_{A}$ currents analysis; (E) Activation curve analysis. Data are expressed as mean \pm SD. ${ }^{* *} p<0.0$ I vs control; ${ }^{*} p<0.05,{ }^{*} p<0.0$ I vs model.

Abbreviations: SSa-H, high saikosaponin A; SSa-L, low saikosaponin A; SSa-M, medium saikosaponin A; VPA, valproic acid.

Most current drugs that inhibit neuronal loss do not have any antiepileptogenic effects. ${ }^{49}$ This observation is consistent with our previous research. ${ }^{50}$

Additionally, we used hippocampal neurons 24 hours after a 3-hour exposure to $\mathrm{Mg}^{2+}$-free solution in this HNC model, which produced SREDs that resembled the discharges of clinical epilepsy, ${ }^{51,52}$ and SSa exerted an anticonvulsant effects. Some anticonvulsants are more effective in certain seizure models than others. For instance, when examining the efficacy on refractory seizures induced by $\mathrm{Mg}^{2+}$-free solution in the immature corticohippocampal formation in vitro, drugs such as valproate suppress epileptiform activity, whereas 
ethosuximide, gabapentin, phenytoin, and topiramate are ineffective in this model. ${ }^{53}$

From further studies in rat brain tissue, we found a significant reduction load of Kv4.2 which may result from decreased levels of Kchip1 during the chronic period of epilepsy both in vivo and in vitro. These decreased Kchip1 levels have been linked to seizure frequency and severity, which was found in other studies as well. ${ }^{54,55}$ A possible explanation for this might be that Kchip1 is prominently expressed in the CNS and may regulate $\mathrm{Kv} 4.2$ in a $\mathrm{Ca}^{2+}$-dependent manner, as well as structural or kinetic activity and the density of cell surface membrane channels. ${ }^{55-57}$ Collectively, SSa has been shown to increase the expression of Kchip1 and Kv4.2 and, thus, to ameliorate epilepsy behavior.

Moreover, to analyze the mechanism of SSa, the Kv4.2mediated $I_{\mathrm{A}}$ currents were tested in hippocampal neurons of the HNC model. A significant downregulation of the Kv4.2mediated $I_{\mathrm{A}}$ current density was found in this study. Several reports have shown a relationship between $I_{\mathrm{A}}$ and epilepsy. For instance, $\mathrm{K}^{+}$currents were abated in hippocampal CA1 pyramidal neuron dendrites in various animal seizure models. ${ }^{22,23,58,59}$ A strong relationship between $\mathrm{Kv} 4.2$ function and epilepsy has previously been reported. ${ }^{42,58,59}$ Animal epileptic models have shown correlations between changes in $I_{\mathrm{A}}$ and reductions in Kv4.2 mRNA expression with increased seizure activity. ${ }^{24,25} \mathrm{~A}$ marked downregulation of classical $I_{\mathrm{A}}$ was observed in dentate granule cells isolated from patients with lesion-associated TLE. ${ }^{60}$ The reduced availability of $\mathrm{Kv} 4.2$-mediated $I_{\mathrm{A}}$ may be caused by a reduction in A-type $\mathrm{K}^{+}$channel density, altered modulation of the channel protein by phosphorylation, or both. ${ }^{42}$ Surprisingly, SSa treatment significantly increased Kv4.2-mediated $I_{\text {A }}$ currents. Therefore, we tentatively suggest that SSa modulates Kv4.2-mediated $I_{\mathrm{A}}$ currents by upregulating Kchip1 and Kv4.2 expressions in the chronic period of rat TLE. Unfortunately, our results lack the direct evidence to confirm the role of Kv4.2-mediated $I_{\mathrm{A}}$ currents and the regulatory effects of SSa. More research in this area should to be undertaken, in order to clarify the association between ion channels and cTLE. In addition, other mechanisms behind the anti-epileptic effects of SSa require further investigation.

\section{Conclusions}

In conclusion, this study showed that decreased Kchip1 and Kv4.2 expression may result in the downregulation of Kv4.2mediated $I_{\mathrm{A}}$ currents, thus producing SRS. However, with SSa treatment, all of these symptoms were ameliorated. Therefore, we hypothesize that SSa modulates Kv4.2-mediated $I_{\mathrm{A}}$ currents by upregulating Kchip1 and Kv4.2 expression in rat cTLE. This work and the previous findings from our research group reinforce the great and unexplored therapeutic potential of SSa for the treatment of cTLE.

\section{Availability of data and materials}

The datasets generated during the current study are available from the corresponding author on request.

\section{Acknowledgments}

This work was supported by the National Natural Science Foundation of China (No. 81373810), the National Natural Science Foundation of China (No. 81703926), the National Natural Science Foundation of Guangdong, China (No. 2015A030313265), the National Natural Science Foundation of Guangdong, China (No. 2017A030310289), the Science and Technology Program of Guangzhou, China (No. 201804010033).

\section{Author contributions}

WX and YH contributed to conception and design the study. YH drafted the article. DN, HNJ and ZZX performed most of the experiments and the animal model studies. YXQ and ZYW analyzed and interpreted data in revised the manuscript. WX finally approval the version to be published. All authors contributed toward data analysis, drafting and revising the paper and agree to be accountable for all aspects of the work.

\section{Disclosure}

The authors report no conflicts of interest in this work.

\section{References}

1. Fisher RS, Acevedo C, Arzimanoglou A, et al. ILAE official report: a practical clinical definition of epilepsy. Epilepsia. 2014;55(4): 475-482.

2. Kakooza-Mwesige A. The importance of botanical treatments in traditional societies and challenges in developing countries. Epilepsy Behav. 2015;52(Pt B):297-307.

3. Engel J. Mesial temporal lobe epilepsy: what have we learned? Neuroscientist. 2001;7(4):340-352.

4. Löscher W, Klitgaard H, Twyman RE, Schmidt D. New avenues for anti-epileptic drug discovery and development. Nat Rev Drug Discov. 2013;12(10):757-776.

5. French JA. Can drug regimen changes prevent seizures in patients with apparently drug-resistant epilepsy? Nat Clin Pract Neurol. 2007; 3(7):364-365

6. Delorenzo RJ, Sun DA, Deshpande LS. Cellular mechanisms underlying acquired epilepsy: the calcium hypothesis of the induction and maintainance of epilepsy. Pharmacol Ther. 2005;105(3):229-266.

7. Harden CL. Evidence that intractable temporal lobe epilepsy is influenced by genetic and environmentally acquired factors. Epilepsy Curr. 2004;4(4):131-132.

8. Gutman GA, Chandy KG, Grissmer S, et al. International Union of Pharmacology. LIII. Nomenclature and molecular relationships of voltagegated potassium channels. Pharmacol Rev. 2005;57(4):473-508.

9. Serôdio P, Kentros C, Rudy B. Identification of molecular components of A-type channels activating at subthreshold potentials. J Neurophysiol. 1994;72(4):1516-1529.

10. Hille B. A K+ channel worthy of attention. Science. 1996;273(5282): 1677. 
11. Hoffman DA, Magee JC, Colbert CM, Johnston D. K+ channel regulation of signal propagation in dendrites of hippocampal pyramidal neurons. Nature. 1997;387(6636):869-875.

12. Pongs $\mathrm{O}$, Leicher $\mathrm{T}$, Berger $\mathrm{M}$, et al. Functional and molecular aspects of voltage-gated K+ channel beta subunits. Ann N Y Acad Sci. 1999; 868:344-355.

13. Benarroch EE. Potassium channels: brief overview and implications in epilepsy. Neurology. 2009;72(7):664-669.

14. Lee H, Lin MC, Kornblum HI, Papazian DM, Nelson SF. Exome sequencing identifies de novo gain of function missense mutation in KCND2 in identical twins with autism and seizures that slows potassium channel inactivation. Hum Mol Genet. 2014;23(13):3481-3489.

15. Singh B, Ogiwara I, Kaneda M, et al. A Kv4.2 truncation mutation in a patient with temporal lobe epilepsy. Neurobiol Dis. 2006;24(2): 245-253.

16. Gardoni F, Mauceri D, Marcello E, Sala C, di Luca M, Jeromin A. SAP97 directs the localization of Kv4.2 to spines in hippocampal neurons: regulation by CaMKII. J Biol Chem. 2007;282(39):28691-28699.

17. Nakamura TY, Pountney DJ, Ozaita A, et al. A role for frequenin, a $\mathrm{Ca} 2+$-binding protein, as a regulator of Kv4 K+-currents. Proc Natl Acad Sci U S A. 2001;98(22):12808-12813.

18. Lugo JN, Barnwell LF, Ren Y, et al. Altered phosphorylation and localization of the A-type channel, Kv4.2 in status epilepticus. J Neurochem. 2008;106(4):1929-1940.

19. Gupte RP, Kadunganattil S, Shepherd AJ, et al. Convergent phosphomodulation of the major neuronal dendritic potassium channel Kv4.2 by pituitary adenylate cyclase-activating polypeptide. Neuropharmacology. 2016;101:291-308.

20. An WF, Bowlby MR, Betty M, et al. Modulation of A-type potassium channels by a family of calcium sensors. Nature. 2000;403(6769):553-556.

21. Ono M, Yoshida A, Ito Y, Nohara T. Phenethyl alcohol glycosides and isopentenol glycoside from fruit of Bupleurum falcatum. Phytochemistry. 1999;51(6):819-823.

22. Juhng KN, Kokate TG, Yamaguchi S, et al. Induction of seizures by the potent $\mathrm{K}+$ channel-blocking scorpion venom peptide toxins tityustoxinK(alpha) and pandinustoxin-K(alpha). Epilepsy Res. 1999;34(2-3): 177-186.

23. Bagetta G, Nisticó G, Dolly JO. Production of seizures and brain damage in rats by alpha-dendrotoxin, a selective $\mathrm{K}+$ channel blocker. Neurosci Lett. 1992;139(1):34-40.

24. Birnbaum SG, Varga AW, Yuan LL, Anderson AE, Sweatt JD, Schrader LA. Structure and function of Kv4-family transient potassium channels. Physiol Rev. 2004;84(3):803-833.

25. Tsaur ML, Sheng M, Lowenstein DH, Jan YN, Jan LY. Differential expression of $\mathrm{K}+$ channel mRNAs in the rat brain and down-regulation in the hippocampus following seizures. Neuron. 1992;8(6):1055-1067.

26. Yu YH, Xie W, Bao Y, Li HM, Hu SJ, Xing JL. Saikosaponin a mediates the anticonvulsant properties in the HNC models of $\mathrm{AE}$ and $\mathrm{SE}$ by inhibiting NMDA receptor current and persistent sodium current. PLoS One. 2012;7(11):e50694.

27. Xie W, Yu YH, du YP, et al. Saikosaponin a Enhances Transient Inactivating Potassium Current in Rat Hippocampal CA1 Neurons. Evid Based Complement Alternat Med. 2013;2013(12):413092:1-10.

28. Racine RJ. Modification of seizure activity by electrical stimulation. II. Motor seizure. Electroencephalogr Clin Neurophysiol. 1972;32(3): 281-294

29. Rigoulot MA, Koning E, Ferrandon A, Nehlig A. Neuroprotective properties of topiramate in the lithium-pilocarpine model of epilepsy. J Pharmacol Exp Ther. 2004;308(2):787-795.

30. Chu K, Jung KH, Lee ST, et al. Erythropoietin reduces epileptogenic processes following status epilepticus. Epilepsia. 2008;49(10):1723-1732.

31. Jung KH, Chu K, Lee ST, et al. Cyclooxygenase-2 inhibitor, celecoxib, inhibits the altered hippocampal neurogenesis with attenuation of spontaneous recurrent seizures following pilocarpine-induced status epilepticus. Neurobiol Dis. 2006;23(2):237-246.

32. Wei X, Meng CX, Shi GJ, Fan SQ, Yu H, Chen WJ. Effect of saikosaponin-a on epileptic seizures in refractory epilepsy rats. $J$ Trop Med. 2012.
33. Xie W, Chen WJ, Meng CX, Liang YC, Yao L, Zheng YH. Effect of saikosaponin a on expression of multidrug resistance protein P-glycoprotein in refractory epilepsy rats. Chin J Exp Trad Med Formul. 2013.

34. González RR, Fernández RF, Vidal JL, Frenich AG, Pérez ML. Development and validation of an ultra-high performance liquid chromatography-tandem mass-spectrometry (UHPLC-MS/MS) method for the simultaneous determination of neurotransmitters in rat brain samples. J Neurosci Methods. 2011;198(2):187-194.

35. Xie W, Cai L, Yu Y, et al. Activation of brain indoleamine 2,3-dioxygenase contributes to epilepsy-associated depressive-like behavior in rats with chronic temporal lobe epilepsy. $J$ Neuroinflammation. 2014;11:41.

36. Blair RE, Deshpande LS, Sombati S, Falenski KW, Martin BR, Delorenzo RJ. Activation of the cannabinoid type-1 receptor mediates the anticonvulsant properties of cannabinoids in the hippocampal neuronal culture models of acquired epilepsy and status epilepticus. J Pharmacol Exp Ther. 2006;317(3):1072-1078.

37. Amarillo Y, de Santiago-Castillo JA, Dougherty K, et al. Ternary Kv4.2 channels recapitulate voltage-dependent inactivation kinetics of A-type $\mathrm{K}+$ channels in cerebellar granule neurons. J Physiol. 2008; 586(8):2093-2106.

38. Maffie JK, Dvoretskova E, Bougis PE, Martin-Eauclaire MF, Rudy B. Dipeptidyl-peptidase-like-proteins confer high sensitivity to the scorpion toxin AmmTX3 to Kv4-mediated A-type K+ channels. J Physiol. 2013;591(10):2419-2427.

39. André V, Rigoulot MA, Koning E, Ferrandon A, Nehlig A. Long-term pregabalin treatment protects basal cortices and delays the occurrence of spontaneous seizures in the lithium-pilocarpine model in the rat. Epilepsia. 2003;44(7):893-903.

40. François J, Koning E, Ferrandon A, Nehlig A. The combination of topiramate and diazepam is partially neuroprotective in the hippocampus but not antiepileptogenic in the lithium-pilocarpine model of temporal lobe epilepsy. Epilepsy Res. 2006;72(2-3):147-163.

41. Linard B, Ferrandon A, Koning E, Nehlig A, Raffo E. Ketogenic diet exhibits neuroprotective effects in hippocampus but fails to prevent epileptogenesis in the lithium-pilocarpine model of mesial temporal lobe epilepsy in adult rats. Epilepsia. 2010;51(9):1829-1836.

42. Bernard C, Anderson A, Becker A, Poolos NP, Beck H, Johnston D. Acquired dendritic channelopathy in temporal lobe epilepsy. Science. 2004;305(5683):532-535.

43. Nishiura T, Marukawa S, Ishida H, Orita M, Abe H. Effects of saikosaponins on hepatic damage induced by halothane and hypoxia in phenobarbital-pretreated rats. J Anesth. 1994;8(1):87-92.

44. Lu CN, Yuan ZG, Zhang XL, et al. Saikosaponin a and its epimer saikosaponin $\mathrm{d}$ exhibit anti-inflammatory activity by suppressing activation of NF- $\mathrm{KB}$ signaling pathway. Int Immunopharmacol. 2012; 14(1):121-126.

45. Chen JC, Chang NW, Chung JG, Chen KC. Saikosaponin-A induces apoptotic mechanism in human breast MDA-MB-231 and MCF-7 cancer cells. Am J Chin Med. 2003;31(3):363-377.

46. Cavalheiro EA, Santos NF, Priel MR. The pilocarpine model of epilepsy in mice. Epilepsia. 1996;37(10):1015-1019.

47. Hamilton SE, Loose MD, Qi M, et al. Disruption of the $\mathrm{m} 1$ receptor gene ablates muscarinic receptor-dependent $\mathrm{M}$ current regulation and seizure activity in mice. Proc Natl Acad Sci U S A. 1997;94(24): 13311-13316.

48. Curia G, Longo D, Biagini G, Jones RS, Avoli M. The pilocarpine model of temporal lobe epilepsy. J Neurosci Methods. 2008;172(2): $143-157$.

49. Löscher $\mathrm{W}$, Potschka $\mathrm{H}$. Role of multidrug transporters in pharmacoresistance to antiepileptic drugs. J Pharmacol Exp Ther. 2002;301(1): $7-14$.

50. Xie W, Meng CX, Shi GJ, Fan SQ, Hong Y, Chen WJ. Effect of Bupleurum saponin A on epileptic seizures in rats with intractable epilepsy. J Trop Med. 2012;10:382-384.

51. Lothman EW, Bertram EH, Stringer JL. Functional anatomy of hippocampal seizures. Prog Neurobiol. 1991;37(1):1-82. 
52. Deshpande LS, Blair RE, Nagarkatti N, Sombati S, Martin BR, Delorenzo RJ. Development of pharmacoresistance to benzodiazepines but not cannabinoids in the hippocampal neuronal culture model of status epilepticus. Exp Neurol. 2007;204(2):705-713.

53. Quilichini PP, Diabira D, Chiron C, Milh M, Ben-Ari Y, Gozlan H. Effects of antiepileptic drugs on refractory seizures in the intact immature corticohippocampal formation in vitro. Epilepsia. 2003;44(11): $1365-1374$

54. Rüschenschmidt C, Chen J, Becker A, Riazanski V, Beck H. Functional properties and oxidative modulation of A-type $\mathrm{K}$ currents in hippocampal granule cells of control and chronically epileptic rats. Eur J Neurosci. 2006;23(3):675-685.

55. Su T, Cong WD, Long YS, et al. Altered expression of voltagegated potassium channel 4.2 and voltage-gated potassium channel 4-interacting protein, and changes in intracellular calcium levels following lithium-pilocarpine-induced status epilepticus. Neuroscience. 2008;157(3):566-576.
56. Strassle BW, Menegola M, Rhodes KJ, Trimmer JS. Light and electron microscopic analysis of KChIP and Kv4 localization in rat cerebellar granule cells. J Comp Neurol. 2005;484(2):144-155.

57. Salvador-Recatalà V, Gallin WJ, Abbruzzese J, Ruben PC, Spencer AN. A potassium channel (Kv4) cloned from the heart of the tunicate Ciona intestinalis and its modulation by a KChIP subunit. $J$ Exp Biol. 2006; 209(Pt 4):731-747.

58. Castro PA, Cooper EC, Lowenstein DH, Baraban SC. Hippocampal heterotopia lack functional $\mathrm{Kv} 4.2$ potassium channels in the methylazoxymethanol model of cortical malformations and epilepsy. JNeurosci. 2001;21(17):6626-6634.

59. Aronica E, Boer K, Doorn KJ, et al. Expression and localization of voltage dependent potassium channel Kv4.2 in epilepsy associated focal lesions. Neurobiol Dis. 2009;36(1):81-95.

60. Beck H, Clusmann H, Kral T, Schramm J, Heinemann U, Elger CE. Potassium currents in acutely isolated human hippocampal dentate granule cells. J Physiol. 1997;498(Pt 1):73-85.

\section{Publish your work in this journal}

Drug Design, Development and Therapy is an international, peerreviewed open-access journal that spans the spectrum of drug design and development through to clinical applications. Clinical outcomes, patient safety, and programs for the development and effective, safe, and sustained use of medicines are the features of the journal, which has also been accepted for indexing on PubMed Central. The manuscript management system is completely online and includes a very quick and fair peer-review system, which is all easy to use. Visit http://www.dovepress.com/testimonials.php to read real quotes from published authors.

Submit your manuscript here: http://www.dovepress.com/drug-design-development-and-therapy-journal 